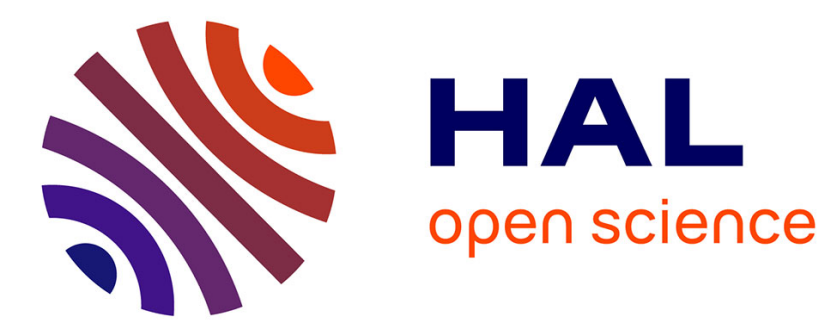

\title{
Suivez le guide! Le tour de France en musique (1852-1870)
}

Isabelle Mayaud

\section{To cite this version:}

Isabelle Mayaud. Suivez le guide! Le tour de France en musique (1852-1870). Culture et Musées, 2014, 23, pp.21-41. hal-01506897

\section{HAL Id: hal-01506897 \\ https://hal.parisnanterre.fr/hal-01506897}

Submitted on 19 Dec 2021

HAL is a multi-disciplinary open access archive for the deposit and dissemination of scientific research documents, whether they are published or not. The documents may come from teaching and research institutions in France or abroad, or from public or private research centers.
L'archive ouverte pluridisciplinaire HAL, est destinée au dépôt et à la diffusion de documents scientifiques de niveau recherche, publiés ou non, émanant des établissements d'enseignement et de recherche français ou étrangers, des laboratoires publics ou privés. 
Citation : Mayaud, Isabelle. «Suivez le guide ! Le tour de France en musique (18521870 », dans Emilie Flon (sous la dir.), «Tourisme et médiations des patrimoines », Cultures et musées, 2014, $\mathrm{n}^{\circ}$ 23, p. 21-41

DOI : https://doi.org/10.4000/culturemusees.1342

Me contacter : isabelmayaud@gmail.com

\begin{abstract}
About the middle of the XIXth century, various agents, in the public as well as in the private field, contribute to determine the area covered by the term "popular songs and poetry" The Fortoul investigation gathers, from that point of view, the most important corpus dealing with the subject. This paper focuses on another group of agents, so far neglected, involved in this public collection. Specialised branch of publication, the printed travel guides give birth to a certain "tourist image" of the "musical France". Generally considered as the main spreader of regional stereotypes that preexisted, the travel guides are considered here as contributing to the framing of the national heritage.
\end{abstract}

Resumen: A mediados del siglo XIX, agentes públicos y privados concurren a determinar el perímetro recubierto por el sintagma "cantos y poesías populares". La investigación Fortoul concentra desde este punto de vista lo esencial de los estudios. Esta contribución se interesa a otro grupo de agentes, contemporáneos a esta colecta pública, hasta entonces poco tomado en cuenta. Fracción especializada en la edición, las guías de viajes impresas entregan una cierta "imagen turística" de la Francia musical. Habitualmente considerados como los difusores privilegiados de los estereotipos regionales preexistentes, las guías de viajes son aquí analizadas como coproductoras de un patrimonio nacional.

Résumé : Au mitan du XIXe siècle, différents agents, publics et privés, concourent à déterminer le périmètre recouvert par le syntagme « chants et poésies populaires ». L'enquête Fortoul concentre sous cet angle l'essentiel des études. Cette contribution s'intéresse à un autre groupe d'agents, contemporains de cette collecte publique, jusqu'alors peu pris en compte. Fraction spécialisée de l'édition, les guides de voyages imprimés délivrent alors une certaine « image touristique » de la France musicale. D'ordinaire considérés comme les diffuseurs privilégiés de stéréotypes régionaux qui leur préexistent, les guides de voyages sont ici envisagés comme des coproducteurs d'un patrimoine national. 


\section{SUIVEZ LE GUIDE !}

\section{LE TOUR DE FRANCE EN MUSIQUE (1852-1870)}

Patrimoine culturel immatériel ${ }^{1} »$, « musiques traditionnelles », « folklore », « chants et poésies populaires » : le survol de la bibliographie relative à l'art musical en France suggère que, depuis le XIX ${ }^{\mathrm{e}}$ siècle, chaque génération ${ }^{2}$ élabore une terminologie spécifique afin de définir un hypothétique objet d'étude univoque : les chants et la musique élaborés par certains habitants des campagnes - et parfois des villes - françaises, transmis par la voie orale et dont la disparition, présentée comme imminente, impose le recueil urgent à des fins de sauvegarde.

Produit d'une illusion rétrospective, cette homologie entre les différents énoncés ne peut être que fallacieuse et historiquement erronée. Les définitions de l'objet «musique », avec adjonction ou non de qualificatif, varient en effet dans la diachronie et la synchronie, en termes à la fois de contours géographiques, temporels et sociaux.

C'est la dimension synchronique qui est ici privilégiée dans l'examen des « chants et poésies populaires ». On se propose de suivre la formation et la diffusion de cet énoncé par et pour différents agents, en France, au milieu du XIX ${ }^{\mathrm{e}}$ siècle. Pionnières sur ces questions, les études sur l'enquête dite Fortoul $^{3}$ se sont multipliées depuis trois décennies ${ }^{4}$. La difficulté à préciser les limites de l'enquête, engagée entre 1852 et 1857 à l'initiative du ministère de l'Instruction publique, nous renvoie précisément à l'indétermination de l'objet de cette étude : «les poésies populaires ». Une commission est ainsi mandatée afin de décrire ce que recouvre le syntagme (Cheyronnaud, 1986 : 12; Laborde, 1998 : 93). Ce vaste recueil est alors inédit de par son ampleur; de par ses visées, il n'est toutefois pas sans précédent. Plusieurs enquêtes s'intéressent, avant cette date, aux productions culturelles ${ }^{5}$, certaines font mention de chants, de danses, etc. L'enquête statistique portée par les préfets à partir de $1801^{6}$ dresse ainsi un premier tableau, certes lacunaire, d'une certaine France musicale. Par ailleurs, de nombreuses collectes sont réalisées par des écrivains romantiques - Nerval, Sand, etc. - ou des associations savantes - l'académie celtique puis la société des antiquaires de France. Ces initiatives privées sont connues, elles ont été plus particulièrement investies par les littéraires (Bénichou, 1970 ; Millot, Vincent-Munnia, Schapira \& Fontana, 2005) et les anthropologues ${ }^{7}$ (Belmont, 1975, 1995 ; Fabre, 2011). Chacune de ces enquêtes est l'occasion d'une définition de «la poésie populaire », les différents agents, individuels ou collectifs, ne s'accordant pas nécessairement sur le contenu de cet objet, ses contours, voire sa validité.

\footnotetext{
${ }^{1}$ En 1972, l'Unesco adopte une convention de sauvegarde du patrimoine mondial qui vise le patrimoine culturel et naturel. En 2003, elle élargit le champ couvert par la convention au patrimoine culturel immatériel.

${ }^{2}$ La génération scientifique contemporaine semble souvent renoncer à l'effort de réflexion et de détermination terminologique et s'accommode de taxinomies musicales dont elle souligne les limites par l'utilisation des guillemets (Buch, 2013 : 46-51).

${ }^{3}$ Du nom du ministre de l'Instruction publique de l'époque : Hippolyte Fortoul (1811-1856).

${ }^{4}$ Voir les mentions qui en sont faites dans : Fulcher (1980) ; De Certeau, Julia \& Revel (1993 [1970]); Cheyronnaud (1986, 1997); Laborde (1998, 2008, 2009); Millot et al. (2005); Charles-Dominique $(2007,2008)$ ; Bécam \& Berthou-Bécam (2010).

${ }^{5}$ Pour exemple, l'enquête publique sur l'état du royaume engagée en 1697 et destinée au duc de Bourgogne s'intéresse aux « hommes : leur naturel vif ou pesant, laborieux ou paresseux, leurs inclinations, leurs coutumes » (Gille, $1980: 29)$.

${ }^{6}$ Marie-Noëlle Bourguet a consacré sa thèse à cette enquête (Bourguet, 1989).

${ }^{7}$ Voir la base de données Bérose, publication en ligne : <http://app. berose.fr/v3/>
} 
Cet article s'intéresse à un autre groupe d'agents contemporains de l'enquête Fortoul, jusqu'alors peu pris en considération dans les études sur les enquêtes musicales menées durant cette période ${ }^{8}$. Fraction spécialisée de l'édition, les grandes collections de guides de voyage se développent en Europe dans les années 1840 et $1860^{\circ}$. Elles mobilisent, en amont et en aval de la production, toute une série d'intervenants : éditeur, imprimeur, écrivain, journaliste, etc. Par ailleurs, elles partagent cette prometteuse part du marché de l'édition avec des publications isolées, insérées dans des réseaux exclusivement locaux. Les guides de voyages sont, avec les agences de voyage qui naissent à la même époque - l'agence Cook est fondée en 1851 -, les principaux intermédiaires de l'industrie touristique européenne qui connaît alors une profonde transformation (Fierro, 1985 : 193 ; Bertho-Lavenir, 1999 : 45 ; Boyer, 2005 : 198 ; Chabaud, Cohen \& Coquery, 2000 : 203). Rouages centraux de cette économie, les guides au XIX ${ }^{\mathrm{e}}$ siècle ont fait l'objet de nombreux travaux, redevables au développement des études de tourisme ${ }^{10}$ et d'histoire culturelle ${ }^{11}$. Plusieurs approches ont été privilégiées, parmi lesquelles nous retenons, car elles informent la présente réflexion, celles qui mobilisent les guides pour écrire l'histoire de la diffusion des stéréotypes culturels dans le contexte de construction nationale (Gerbod, 1991, pour le cas français), l'histoire des savoirs sur l'espace et l'histoire du patrimoine matériel - la culture architecturale (Toulier, 2010 ; Vajda, 2010 - ou immatériel - la gastronomie (Csergo \& Lemasson, $\left.2008^{12}\right)$.

Les guides de voyage constituent une source précieuse de localisation des différents lieux de musique en France au XIX ${ }^{\mathrm{e}}$ siècle. Ils gagnent, en outre, à être étudiés pour eux-mêmes : produits d'intermédiation culturelle, ils sont aussi le produit d'une culture éditoriale spécifique. Afin de mener cette étude, nous avons formé un double échantillon ${ }^{13}$, déclinant deux échelles spatiales et temporelles : la France - préférentiellement rurale - entre 1852 et 1857, les Pyrénées entre 1852 et 1870. Le développement qui suit porte ainsi tant sur les contenus que sur les producteurs de ces guides et vise à préciser et expliciter le tableau contrasté que ce groupe d'agents dresse de la France musicale au milieu du XIX ${ }^{\mathrm{e}}$ siècle.

\section{LA LUTTE DES CLASSES MUSICALES}

Alors même que les correspondants du ministère de l'Instruction publique recueillent par dizaines les chants et poésies populaires dans les campagnes, c'est une autre France musicale qui nous est donnée à voir dans les guides imprimés de voyage pour la période 1852-1857. De fait, si les occurrences de la musique en général sont nombreuses dans notre échantillon, celles ayant spécifiquement trait à la France rurale sont relativement rares dans notre corpus.

\footnotetext{
${ }^{8}$ Luc Charles-Dominique fait seulement mention de quelques guides pour l'époque moderne, et ce, afin d'illustrer un «éveil ancien d'un intérêt de plus en plus marqué pour les cultures françaises de l'oralité » (Charles-Dominique, $2008: 128$ ).

${ }^{9}$ Les premiers guides de voyage imprimés datent du XVI ${ }^{\mathrm{e}}$ siècle. Les collections de guides apparaissent dans la première moitié du XIX ${ }^{\mathrm{e}}$ siècle chez différents éditeurs : Murray en 1836 pour la Grande-Bretagne, Baedeker en 1846 pour l'Allemagne, Hachette en 1853 pour la France - la collection des guides Joanne est vendue par Hachette à partir de 1857 - (Vajda et al., 2009 : 255). Voir Guilcher (2011) pour une revue des principales collections (Murray, Baedeker, Joanne) et de quelques autres plus modestes (Langlois, Galignanti, Leigh, Chaix, Garnier, Coghlan, Bradshaw, etc.).

${ }^{10}$ Depuis les années soixante-dix, le « tourisme » s'est constitué en objet de recherche investi par différentes disciplines scientifiques. Pour une bibliographie commentée des études portant sur l'histoire du tourisme, voir notamment : Réau \& Poupeau (2007 : 4-13) ; Cousin \& Réau (2009) ; Venayre (2012 : 407-455, chap. XIV : « Les logiques du tourisme »).

${ }^{11}$ Pour une bibliographie commentée des études en français sur les guides de voyage, voir Cohen \& Toulier (2011) et Cohen (2011).

${ }^{12}$ Pour une bibliographie critique sur les guides, voir Cohen \& Toulier (2011).

${ }^{13}$ La délimitation du corpus étudié résulte de plusieurs opérations de tris opérées à partir du catalogue général en ligne de la Bibliothèque nationale de France. Une première sélection pour la période 1852-1857 a permis d'isoler une soixantaine de guides parus en France sur l'espace français durant cette période. Un second échantillon regroupe vingt-deux guides des Pyrénées, publiés entre 1852 et 1870.
} 


\section{DES INTÉRIEURS URBAINS ET BOURGEOIS}

À la frontière urbanité/ruralité musicale induite et véhiculée par l'enquête Fortoul, les guides imprimés opposent une division territoriale alternative. La fracture culturelle, telle que construite par cette production, s'ancre en effet dans des caractéristiques principalement sociales qui se déploient dans l'espace de la ville. Au fil des pages se dessine ainsi une cartographie des différents lieux de musique urbains. D'une ville à l'autre sont énumérées - de manière non systématique mais récurrente - : les adresses des professionnels - facteurs, marchands, professeurs -, les écoles de musique et les salles de concert. Les informations dispensées concernant les professionnels sont d'ordre pratique. Le guide fait ici office d'annuaire ${ }^{14}$, outil de localisation et de description succincte, qui, les manufactures de piano, les facteurs et les fabricants parisiens (Paris à la main, $1855: 3,17,21,43$ ); qui, les professeurs de musique de Moutiers (Collet, 1853), listés en appendice entre « quincaillerie-mercerie » et « brasserie ». Les guides répertorient également les écoles de musique : sont ainsi indiquées les succursales du conservatoire de Metz (Terquem, 1854 : 58) et de Toulouse (Gimet, 1857 : 98). Les salles de concert, enfin, font l'objet d'une attention renouvelée, la question de la qualité du son étant souvent centrale. La salle de concert du cercle musical de Lyon installée dans une ancienne église est ainsi conforme aux règles de l'acoustique (Chambet, 1853 : 165-166), tandis que la salle Franklin à Bordeaux « laisse quelque peu à désirer pour la sonorité » (Lamothe, 1856 : 157). Apparaissent donc, dans ces guides, un certain nombre de lieux de musique propres au XIX ${ }^{\mathrm{e}}$ siècle qui ponctuent l'espace urbain, lieux de consommation culturelle pour les amateurs de musique ou de formation pour les apprentis musiciens et musiciennes.

\section{AUX GUINGUETTES POPULAIRES DES ENVIRONS DE PARIS}

Aux antipodes de la salle de musique se trouve la place publique, espace extérieur, ouvert à la libre circulation de tous, qui accueille la musique militaire - tous les jours sur la place Bellecour de Lyon (Chambet, $1853: 51$ ) - ou les manifestations musicales liées aux grandes foires : la fête de SaintCloud qui s'étale sur trois semaines en septembre (Guide dans les environs de Paris, 1855 : 15 ; Villiers, $1855: 11-12$ ) et la fête des loges à Saint-Germain qui dure trois jours, en septembre également (Villiers, 1855 : 51 ; Joanne, $1856: 351$ ). La fête des loges en 1855 est ainsi présentée : « Ces paisibles retraites sont envahies par une immense quantité de boutiques, de baraques, de bateleurs, de tentes, de bals et de cuisines en plein vent. Pendant trois jours et trois nuits on mange, on boit, on danse, on s'amuse. Les cris de joie se mêlent aux accords de l'orchestre et aux détonations des tirs à la carabine, que domine magistralement la symphonie criarde des mirlitons, instrument si apprécié sur les rives de la Seine. Ces trois jours passés, tout rentre dans le silence. » (Guide dans les environs de Paris, 1855 : 51.$) \mathrm{Au}$ raffinement et au doux son des intérieurs urbains s'oppose donc le bruit de cet espace suburbain ou « campagne » des ouvriers ${ }^{15}$. Cafés-restaurants ou guinguettes, tels sont leurs principaux lieux clos de sociabilité. Les qualificatifs ne manquent pas pour déprécier les musiques qui y sont produites et les mœurs qui les accompagnent. Romainville, cet «affreux village », ne présente « qu'une longue succession de cabarets, de restaurants et de bals ${ }^{16}$ champêtres » (Joanne, $\left.1856: 103\right)$. Robinson, autrefois si paisible, est devenue une ville de guinguettes qui compte même « un café-concert au milieu des bois ! », dont se désole l'auteur du guide songeant aux «promeneurs avides de joies plus douces et plus choisies » (Villiers, 1855 : 56). La musique des campagnes ouvrières fait littéralement irruption dans un univers social qui n'est pas le sien.

\footnotetext{
${ }^{14}$ Pour une définition de « l'annuaire », voir Fouché, Péchoin \& Schuwer (2002: 101).

${ }^{15} \mathrm{Au}$ XIX ${ }^{\mathrm{e}}$ siècle, on emploie indistinctement « environs de Paris » et « campagne » pour désigner cette zone située à environ une lieue autour de la ville (Csergo, 2004 : 16). Voir Rabault-Mazières (2000 : 317-327, chap. 23 sur « Les environs de Paris dans les guides touristiques du XIX ${ }^{\mathrm{e}}$ siècle »).

${ }^{16}$ Sur les bals et la ségrégation sociale dans les lieux de danse, voir Gerbod (1987).
} 
Une remarque de Léonce de Lamothe (1812-1874) suggère que ces incursions, par ailleurs isolées au sein de notre corpus, résulteraient d'injonctions éditoriales : « Nous nous fussions volontiers bornés à parler des plaisirs sérieux, de ceux où l'esprit surtout trouve un aliment. Mais il faut obéir à un sévère éditeur, et parler un peu de tout, alors même qu'on l'ignorerait. Entrons donc dans les lieux de plaisance ; et, à l'instar de la bonne société du lieu, regardons à travers les vitres ce qui s'y passe. » (Lamothe, 1856 : 158.) Ce notable et érudit Bordelais nous invite à questionner la nature du lectorat de ces guides. Ceux-ci s'adressent, par définition, aux voyageurs, auxquels ils proposent de faciliter l'accès, matériel et symbolique, aux régions qu'ils décrivent. Or, si l'essor du tourisme au XIX ${ }^{\mathrm{e}}$ siècle est associé à l'apparition du temps libre et au développement des loisirs (Corbin, 2009 : 11), ceux-ci demeurent le privilège d'une élite, aristocratique et bourgeoise. Les descriptions des villes thermales, qui concentrent une multitude d'équipements, illustrent bien les pratiques culturelles du public visé par ces ouvrages. Le divertissement occupait en effet une place importante dans ces centres (Rauch, 2001 : 34-39 ; Penez, 2005 : 181-238), la musique se taillant une part de choix dans le panel de distractions proposées aux curistes. Le guide de Boulogne-sur-Mer (Brunet, 1856) en fournit sans doute l'exemple le plus complet. À la fin de l'ouvrage se trouve consignée la liste des professionnels : marchands de musique, loueurs de pianos, accordeurs et réparateurs de pianos, professeurs de danse et professeurs de musique - avec le détail des enseignements : le piano principalement, mais aussi le chant, le violon, la guitare, l'harmonie, l'accompagnement, la composition, la clarinette et le cornet à piston. On signale en outre une société philharmonique - en fin d'ouvrage - et une école communale de musique (ibid. : 87-88). Par ailleurs, le guide livre une description minutieuse de la vie musicale dans cette ville thermale. L'établissement des bains de mer "se compose : d'un grand salon de réunion et de danse : d'une salle de musique, exclusivement réservée aux dames et où elles ont toujours un piano à leur disposition » (ibid. : 123). Enfin, «Boulogne est la ville privilégiée de toutes les célébrités musicales, qui semblent s'y donner rendez-vous. Les concerts s'y succèdent sous mille formes » et dans diverses salles (ibid. : 125).

On pourrait ainsi multiplier les exemples à l'envi de lieux de musique socialement situés (Bödeker, Veit \& Werner, 2002 : 244) : à Vichy, « la reine des villes d'eaux » (Penez, 2005 : 87), se produisait l'orchestre de Strauss (Castanié, 1857 : 9), pendant qu'aux Eaux-Bonnes on jouait du piano, pratique distinctive pour les femmes dans ces années 1850 (Chaline, $1983: 188$ ). Ce panorama de lieux et de pratiques urbaines, en intérieur, dans des lieux assujettis à des droits d'entrée divers, informe sur la clientèle des prestataires touristiques - transport, hébergement - et de leurs intermédiaires. Le loisir s'ouvre au milieu du XIX ${ }^{e}$ siècle à une nouvelle frange de la population, ce que regrettent d'ailleurs certains ${ }^{17}$, mais cet élargissement social du groupe des bénéficiaires d'un temps de « vacance » (Rauch, 2001 [1996]) demeure très relatif et ne concerne ni les ouvriers ni les paysans.

\section{LA POÉSIE NATURELLE DES CIMES}

Se pose donc la question de la place de cette classe sociale dans les guides de voyage. Puisqu'elle n'en constitue pas le public, pourquoi ses lieux de sociabilité musicale sont-ils indiqués ? La présentation qu'en donne Lamothe plus haut suggère que « le peuple » n'intervient pas comme sujet mais comme objet dans cette part spécifique du marché de l'édition. Objet de curiosité, il l'est à Boulogne-sur-Mer lorsqu'à l'occasion de fêtes champêtres, les touristes étrangers se mêlent à ses quadrilles - cas unique et explicite de mixité sociale dans notre corpus, soulignons qu'il s'agit de touristes anglais (Brunet, 1856 : 127). Il l'est en outre dans les guides des Pyrénées qui, sur la période 1852-1857, dévoilent une authentique société paysanne animée de chants ancestraux, présentant une définition du «populaire » conforme aux critères adoptés alors par les correspondants de l'enquête Fortoul.

\footnotetext{
${ }^{17}$ « Mais Angers paraît n'avoir plus besoin d'une Maison des Arts [...] est-ce donc depuis que tout le monde apprend la musique. » (Lachèse, 1853 : 125.)
} 
« Demandez au premier Aspois qu'il vous dise La haüt sus la mountagnes, et vous aurez une citation supérieure à la mienne, car elle aura de plus toute la valeur de ce que l'on appelle la couleur locale : La haüt sus la mountagnes, u pastou malhurous [...]. » (Landrin, 1853 : 37-38.) « Chant national de la vallée [...] poésie naturelle d'un peuple pasteur »(Landrin, 1853 : 37-38), La haüt sus la mountagnes, composé par le poète béarnais Cyprien Despourrins (1698-1759), a fait l'objet, au cours de la première moitié du XIX ${ }^{\mathrm{e}}$ siècle, de plusieurs rééditions - par Émile Vignancour puis Frédéric Rivarès notamment (Bénichou, 1970 : 116). Les guides se font l'écho et prolongent cet engouement éditorial pour une production culturelle présentée comme essentiellement transmise par voie orale. Les mentions d'instruments de musique sont par ailleurs très rares dans les descriptions - un tambourin dans le Guide des étrangers aux eaux thermales des Hautes-Pyrénées... (1857 : 22). Tout comme les agents de l'enquête Fortoul, les auteurs des guides de voyage mettent ainsi l'accent sur la parole, le plus souvent le patois, parfois accompagné de la traduction en français ${ }^{18}$. Ils partagent en outre l'inquiétude des représentants du ministère de l'Instruction publique quant au progrès de la civilisation et à ses multiples avatars : "Combien de temps auront-elles encore cet attachement aux traditions des aïeux ? C'est ce qu'il est impossible de dire. Déjà les antiques coutumes sont battues en brèche sur bien des points. Chaque jour les jeunes gens qui reviennent du service militaire, les ouvriers étrangers qu'attirent les travaux de construction à Bonnes, les riches voyageurs malades ou curieux, qui abondent pendant la saison des eaux, modifient par leur contact répété, les idées et les goûts des habitants d'Ossau, et tendent à faire disparaître les différences qui existent encore entre eux et le reste des Français. Ainsi tout se nivelle, tout s'aplanit, tout se rapproche. » (Audin, 1853 : 253.) Le paradoxe, très relatif ${ }^{19}$, c'est que l'économie dont relèvent ces guides de voyage, le tourisme, contribue directement, ce qu'expose ici Audin, à accélérer la disparition programmée de ce patrimoine culturel.

\section{UN PATRIMOINE NATIONAL COMMUN}

Primitifs de l'intérieur (Burguière, 2003 ; Laborde, 2009 ; Fabre, 2011), les montagnards sont porteurs d'une tradition, encore intacte, qui s'ancre dans un glorieux passé national. Quelques grandes figures royales sont ainsi mobilisées afin de faire le lien entre la région et la nation, et entre le passé et le présent. Les pasteurs prennent part aux fastueuses festivités organisées par Marguerite de Navarre (1492-1549) : «Pendant les fêtes que donnait Marguerite à Cauterets, les montagnards pyrénéens avaient composé et chantaient les Trois Colombes de Cauterets, cette chanson si plein de naïveté et de grâce. » Suivent la chanson en patois et sa traduction en français en note de bas de page (Chaix, 1853 : 106-107). Louis XV put apprécier, nous dit-on, les chants du poète Despourrins : " C'est lui qui fit ces jolies chansons que vous entendrez à chaque pas, que vous ne vous lasserez pas d'écouter, et que Jeliotte et Garat, tous deux nés dans les Pyrénées, répétèrent, l'un à la cour de Louis XV, l'autre dans maints concerts. » (Landrin, 1853 : 37.) Le national et le local sont donc ici « désignés comme parfaitement solidaires » (Thiesse, $1997: 1$ ) ; la musique des montagnards s'inscrit naturellement dans l'histoire culturelle de la nation. Ici encore, le chant du peuple demeure toutefois un « objet » : objet de distraction traditionnelle de la maison royale, il devient un objet de divertissement pour les élites de l'après 1789 . A contrario de la figure de l'ouvrier qui s'abandonne dans les guinguettes des faubourgs, le montagnard apparait jovial et innocent - un bon sauvage en somme. Autrefois repoussé et combattu ${ }^{20}$, stigmatisé comme un être dénué de culture, a fortiori de culture musicale, il est à présent convoité. Ce renversement de perspective touche alors une petite partie de la chaîne des Pyrénées, « les monts affreux » (Cubero, 2009 : 75) se voient requalifiés pour devenir des espaces de vie authentiques, emblèmes d'une nation dont ils contribuent à asseoir l'anciennetée ${ }^{1}$.

\footnotetext{
${ }^{18}$ Sur le lien entre musique et langue dans une perspective de construction de l'identité nationale, voir Thiesse (2001).

${ }^{19}$ Sur l'ambivalence de ces discours, voir De Certeau, Julia \& Revel (1993) et Fabre (2011).

${ }^{20}$ Voir pour exemple l'enquête de l'abbé Grégoire engagée en 1790.

${ }^{21}$ Les nouvelles nations s'imaginent d'emblée comme anciennes (Anderson, $1983: 14$ ).
} 
Plusieurs éléments concourent à la fabrique de cette personnalité régionale singulière et in fine à la transformation « du pittoresque en patrimoine » (Becker-Jeanjean ${ }^{22}, 1999$, cité dans Cousin \& Réau, 2009 : 14). «L'invention » des régions françaises dans la première moitié du XIX siècle est un phénomène connu, développé dans une série de monographies ${ }^{23}$. Serge Briffaud et José Cubero ont spécifiquement travaillé sur la région pyrénéenne en ce sens ${ }^{24}$. Des études sur le tourisme thermal (Gerbod, 1983, 1985 ; Penez, 2005) ont également contribué à la connaissance des transformations économiques propres aux stations pyrénéennes durant cette période. Dans la tradition du voyage romantique plébiscitant la montagne qui, d'hostile et dangereuse, devient plaisante et majestueuse (Boyer, 2005 : 209), les guides accompagnent et alimentent ainsi un engouement pour le voyage, dans les Alpes et les Pyrénées en particulier. Le tourisme thermal s'est précocement développé dans la région pyrénéenne et il profite, au milieu du siècle, des efforts, publics et privés, consentis en matière d'infrastructures de transports. Relativement à d'autres régions, le développement touristique des Pyrénées est ainsi très avancé dans les années cinquante ; à titre indicatif, le guide Richard connaît onze réimpressions entre 1834 et 1856 (Briffaud, 1994 : 350). Par ailleurs, la culture régionale, très tôt investie par les élites locales ${ }^{25}$, devient un enjeu géopolitique dans les années cinquante : la France et l'Espagne négocient alors leur frontière pyrénéenne (Sahlins, 1989). Ces différents éléments - la précocité du développement touristique, l'intérêt des érudits pour la culture locale, le contexte géopolitique, et dans une certaine mesure aussi l'éloignement avec Paris - contribuent à faire de cette région un espace pionnier en termes d'imposition d'une « image touristique ».

Les chants populaires occupent d'abord une place mineure dans ces portraits régionaux. Leurs occurrences dans les guides des Pyrénées ne sont pas systématiques en début de période. Le guide Chaix de 1853 dresse ainsi, dans sa préface, un long portrait des Basques, dans lequel il évoque en particulier leur langue mais ne fait aucune mention de chants ou de musique (Chaix, 1853 : XIII-XXXII). Vingt ans plus tard, les chants sont devenus incontournables dans les descriptions du peuple des montagnes, mais aussi des côtes. Le spectacle de la culture pittoresque s'étend alors autour de Biarritz - lieu de villégiature de la famille impériale - et sur la côte méditerranéenne. À Collioure, « les chants, la musique, les feux de joie, tout cet ensemble si pittoresque offre un spectacle étrange, imposant et qui mérite d'être vu» (Lacroix, $1865: 128)$.

\section{UNE PRESCRIPTION DE CONCERT ?}

Le rôle des guides de voyage dans la construction identitaire en France au XIX ${ }^{\mathrm{e}}$ siècle, bien que difficile à mesurer, est certain ; leur importance dans la diffusion de représentations stéréotypées est ainsi souvent soulignée. En ce sens, les guides constituent un outil de médiation culturelle qui contribue à imposer une stratification sociale des goûts musicaux, telle que nous venons de la décrire, qui oppose la musique de l'élite à celle du peuple. Objet de curiosité pour Léonce de Lamothe, objet de spectacle pour Joseph Lacroix : la culture musicale populaire se constitue en objet de plaisir dont se repaissent les élites. Une analyse plus fine du corpus des guides des Pyrénées publiés au cours de la période 18521870 invite toutefois à formuler des conclusions plus nuancées. En effet, de même que « le peuple » ne se décline pas au singulier - il y a au moins deux peuples dans ces guides : celui des villes et celui des campagnes -, de même l'unité sociale de « l'élite » peut être mise en question, et avec elle celle de cette production imprimée.

\footnotetext{
${ }^{22}$ Becker-Jeanjean (Caroline). 1999. Les Récits illustrés de voyages pittoresques publiés en France entre 1770 et 1855. Th. Doct. : histoire, École nationale des Chartes, Paris.

${ }^{23}$ Depuis trois décennies, de nombreux ouvrages ou articles s'intitulent ainsi « l'invention » ou « la fabrique » de la Bretagne, de la Normandie, etc. Voir l'article pionnier de Bertho (1980).

${ }^{24}$ Voir également Laborde (2004) sur l'invention de la tradition basque.

${ }^{25}$ Cet investissement est toutefois variable suivant les régions. Dans les départements pyrénéens, les élites se mobilisent d'emblée (voir la carte de France de l'état de l'enquête en 1806, Bourguet, 1989 : 130).
} 
La publication du guide Richard ${ }^{26}$ sur les Pyrénées en 1834 marque un tournant dans l'histoire des guides de cette région : pour la première fois, un guide pour les voyageurs n'émane pas d'un autochtone (Briffaud, 1994 : 349). Ce qui constitue l'exception alors va bientôt devenir la norme : dans les décennies suivantes, deux types principaux d'éditeurs se partagent le marché. Les éditeurs parisiens mobilisent principalement des ressources, matérielles et humaines, étrangères aux régions qu'ils décrivent, pour développer leurs grandes collections de guides. Les imprimeurs et les éditeurs locaux publient des écrits d'auteurs souvent originaires de la région, résidents permanents ou temporaires. Entre 1853 et 1857 paraissent ainsi six guides : trois sont imprimés par le Tarbais Telmon (Abadie, 1853 ; Landrin, 1853 ; Guide des étrangers aux eaux thermales..., 1857), les trois autres sont édités à Paris un guide Chai ${ }^{27}$ et deux rééditions du guide Richard. Cette partition Paris/province perdure ensuite : les éditions Hachette ${ }^{28}$ et les éditions Garnier ${ }^{29}$ publient à leur tour un guide sur la région pyrénéenne, tandis que paraissent des ouvrages hétérogènes qui émanent de diverses petites maisons d'édition du sud-ouest de la France. Plusieurs centaines de kilomètres séparent ces pôles de production et dans l'intervalle, ce sont autant de manières différentes de travailler et de fabriquer des ouvrages qui s'élaborent.

Des écarts matériels s'observent, notamment dans le format choisi : in-16 ou in-18 pour les grandes collections, in- 8 ou même in- 4 parfois pour certains ouvrages produits par les imprimeries locales - deux guides-albums publiés en 1853 sont au format in-4. Les manières de travailler à l'écriture de ces guides varient également d'un pôle à l'autre. Les grandes collections de guides parisiennes mettent en place des dispositifs informationnels méthodiques et transférables. La série des guides Joanne édités par Hachette, par ailleurs bien étudiée, mobilise pour chacun de ses titres une équipe de travail (Nordman, 1986 : 534-535). Ces guides, dont le journaliste Adolphe-Laurent Joanne (1813-1881) est le maître d'œuvre et parfois l'auteur - des guides de montagne en particulier -, sont informés par des recherches livresques et des collaborateurs occasionnels qui résident dans les espaces décrits.

Les guides composés par les érudits locaux répondent, quant à eux, à des logiques différentes. Les préfaces et autres paratextes renseignent souvent sur les conditions de la rédaction de ces ouvrages. L'abbé Adolphe Guilhou profite de ses trois cures aux Eaux-Bonnes pour mener des observations qui viennent nourrir un guide dont il forme le projet à cette occasion. L'ouvrage, rédigé du point de vue du " poète-historien », s'attache à montrer la réalité de la station d'Eaux-Bonnes dans toute sa beauté (Guilhou, 1858 : 10). Ces guides du second type, fruit d'un labeur individuel, sont ainsi souvent tributaires de la trajectoire personnelle et professionnelle de leurs auteurs. Ces derniers appartiennent tous à l'élite sociale, résidents permanents, parfois curistes, résidents temporaires donc, mais pour de longues périodes ${ }^{30}$ Le cas d'Adolphe Guilhou, ecclésiastique et ancien professeur de belles-lettres, n'est pas isolé. Roger comte de Bouillé vient à Pau en 1865 pour les besoins de santé de sa femme et parcourt les Pyrénées avec ses trois filles (Lévy, 2000 : 127-128). Le comte Henry Russel (1834-1909), aristocrate irlandais par son père et gascon par sa mère, s'installe dans la région et initie le pyrénéisme - par analogie avec l'alpinisme. Originaire des Pyrénées, Émilien Frossard (1802-1881) est pasteur. Avec Frédéric Soutras (1815-1884), professeur de lettres au collège de Bagnères-de-Bigorre, il anime la société Ramond, une société savante locale. Adolphe Bourdeillette est docteur, J. A. Lescamela imprimeur. À cette élite de notables locaux qui, conformément aux codes distinctifs de leur classe,

\footnotetext{
${ }^{26}$ Sur les guides Richard, voir Guilcher (1997).

${ }^{27}$ Les premiers guides édités parNapoléon Chaix sont des guides ferroviaires. Ils comptent parmi les premières grandes collections de guides lancées en France, mais ils ne parviennent pas à s'imposer sur le marché, le refus d'Hachette de distribuer ces ouvrages dans les bibliothèques de gare n'étant pas étranger à cet échec (Guilcher, 2011).

${ }^{28}$ Sur les guides Joanne publiés par Hachette, on renvoie à l'article de Daniel Nordman (1986) et à l'ouvrage d'Hélène Morlier (2007).

${ }^{29}$ Sur les guides Garnier, voir Guilcher (1994).

${ }^{30}$ Les renseignements biographiques sont extraits de Vapereau (1893), Balteau (1932), Thomas (1999), Lévy (2000), Béraldi (2011).
} 
s'adonnent aux travaux d'érudition pendant leurs temps libres (Chaline, $1983: 188$ ), on peut opposer une élite entrepreneuriale parisienne qui investit avec succès dans l'industrie du livre, la vente des guides Joanne ayant, pour exemple, largement contribué à la fortune de l'éditeur Louis Hachette.

\section{UNE PRODUCTION ÉDITORIALE CONTRASTÉE}

À cette diversité des élites impliquées dans le processus de production de guides correspond une hétérogénéité de la production elle-même. Le tableau général de la France musicale que nous donnent à lire ces ouvrages, s'il traduit et véhicule une opposition binaire peuple/élite, mérite ainsi d'être affiné. L'examen des différentes variantes dans la description de la ville d'Eaux-Bonnes délivre un aperçu de la complexité des positions discursives. Audin donne une très longue description du « Dimanche aux Eaux-Bonnes » (Audin, 1853 : 240-255), détaillant les « Jeux, Courses et Danses des Ossalais ». Il prend soin de résumer sa position, laissons-lui la parole : «L'œil est ici beaucoup plus flatté que l'oreille, car rien n'est moins harmonieux que ces chants dont retentissent les vallons des Eaux-Bonnes. » (245.) Seules les danses des villageoises - les Ossalais ayant d'après lui le sens du rythme - trouvent grâce à ses yeux. L'abbé Guilhou porte un regard moins catégorique, mais il fait lui aussi peu de cas de ces chants : «C'est la musique simple et peu avancée des tribus montagnardes de l'ancienne Gaule, et qui ne s'est pas beaucoup développée, ni modifiée, en traversant les âges. " Reste que malgré leurs divergences, Guilhou comme Audin encouragent tous deux les touristes à l'excursion, ou partie de campagne ; suivant la tradition aristocratique, les curistes sont invités à se distraire ponctuellement aux alentours des stations (Gerbod, 1985 : 149). Par extension, le paysage naturel pittoresque inclut désormais le spectacle de la culture ; les danses et les chants des montagnards devenant ici une «choseà-voir » (Gritti, 1967 : 51). Pour leur part, Frédéric Soutras et Joseph-Bernard Abadie, notables locaux, ne font aucune mention des environs champêtres et se concentrent exclusivement sur la station d'EauxBonnes. Ils mettent l'accent sur des pratiques élitistes urbaines, le tableau de la vie musicale étant conforme à celui présenté plus haut : "La vie thermale y est peu bruyante, ce qui n'empêche pas néanmoins que l'on trouve à Bonnes de charmantes distractions ; les artistes parisiens y suivent la foule élégante. » (Soutras, 1858 : 99.) Le «populaire » est absent des descriptions délivrées par ces auteurs sur la station d'Eaux-Bonnes; les musiciens ici sont des « artistes », parisiens qui plus est.

Le développement qui précède invite ainsi à soulever la question des divergences de positions des agents suivant leurs caractéristiques sociales et spatiales. Cet aspect a déjà été pointé pour la période antérieure par Marie-Noëlle Bourguet qui, dans son étude sur l'enquête de 1801, montre comment la définition de la culture, les fêtes et les danses paysannes en l'occurrence (Bourguet, 1989 : 207-209), est un enjeu de luttes entre les différentes élites locales. Pour la période postérieure, Saskia Cousin a développé, à travers le cas du tourisme à Loches $(2008,2011)$, un exemple de connivence d'intérêts entre des élites régionales et nationales, leur alliance produisant des effets délétères sur les populations locales. L'histoire de ces jeux de positions et de positionnements, esquissée ici pour les élites du milieu du XIX ${ }^{e}$, et le savoir contrasté qu'elles produisent et diffusent sur la France musicale, reste quant à eux à écrire.

\section{CONCLUSION}

Au terme de l'examen de ce corpus, un certain nombre de conclusions peuvent être formulées. Plusieurs définitions du «populaire » coexistent autour des années cinquante. Dans un premier temps, la plupart des guides de voyage passent sous silence les chants et poésies populaires du type de ceux alors collectés dans le cadre de l'enquête Fortoul. Pour nos auteurs, la France musicale est essentiellement urbaine et bourgeoise. Les poésies et les chants populaires, au milieu du XIX ${ }^{\mathrm{e}}$ siècle, ne sont donc pas d'emblée pittoresques, ils le deviennent au terme d'un processus de production savante auquel prend part la chaîne d'édition des guides. Le cas des Pyrénées permet ainsi d'observer cette fabrique d'une image touristique régionale, les chants populaires y trouvant une fonction d'objet de spectacle pour les élites. À cette date, la logique d'appartenance de classe préside ainsi à la logique d'appartenance régionale. Le «peuple », d'où qu'il soit, est constitué en objet - objet de curiosité, de 
distraction et de savoir. La stratification des pratiques et des goûts musicaux conforte ici la ségrégation sociale.

Toutefois, l'hétérogénéité des définitions d'une certaine culture populaire diffusées par ces guides doit être soulignée. Ce constat invite à nuancer une analyse classique qui fait des guides de voyage des véhicules privilégiés de « stéréotypes » (Chabaud, Cohen \& Coquery, $2000: 20$ ). Si leur rôle de médiateur culturel est avéré, leur intervention dans le processus de production patrimoniale doit être mesurée. Bien plus qu'un motif récurrent des descriptions touristiques, la définition du patrimoine, équivoque du fait même de la pluralité sociale des agents qui concourent à son invention et à sa pérennisation, apparaît en effet l'enjeu même de ces guides. Intermédiaires touristiques, ils participent activement à la construction sociale des frontières symboliques ${ }^{31}$ qui discriminent plusieurs mondes de musique.

I. M.

EHESS Simmel - Université Paris 8 Labtop

Manuscrit reçu le : 5 mars 2013

Version révisée après expertise, reçue le : 9 décembre 2013 Article accepté pour publication le : 17 décembre 2013

\section{RÉFÉRENCES BIBLIOGRAPHIQUES}

Abadie (Joseph-Bernard). 1853. Guide-album aux eaux des Pyrénées. Vallée d'Ossau, par Abadie (de Sarrancolin). Eaux-Bonnes, Eaux-Chaudes, Ogeu. Tarbes : Telmon.

Anderson (Benedict). 1983. L'Imaginaire national. Réflexions sur l'origine et l'essor du nationalisme. Paris : La Découverte [traduit par P.-E. Dauzat].

Audin (Jean-Marie-Vincent). 1853. Guide du voyageur aux Pyrénées : Itinéraire pittoresque et artistique du géologue, de l'homme du monde et du malade aux bains des Hautes et BassesPyrénées, des Pyrénées Orientales et de la Haute-Garonne, par Richard (J.-M.-V. Audin). Paris : Maison.

Auteur inconnu. 1855. Paris à la main, guide des voyageurs dans la capitale et aux environs. Paris : Impr. de A. Delcambre.

Barth (Fredrik). 1969. Ethnic Groups and Boundaries: The Social Organization of Culture Difference. Boston : Little, Brown and Company.

Bécam (Didier) \& Berthou-Bécam (Laurence). 2010. L'Enquête Fortoul, 1852-1876 : Chansons populaires de Haute et Basse-Bretagne. Rennes : Dastum.

Becker-Jeanjean (Caroline). 1999. Les Récits illustrés de voyages pittoresques publiés en France entre 1770 et 1855. Th. Doct. : histoire, École nationale des Chartes, Paris.

\footnotetext{
${ }^{31}$ Sur la construction sociale des frontières, on renvoie au livre pionnier de Barth (1969). Pour un examen critique de cette notion de frontière dans les sciences humaines et sociales, voir Jeanpierre (2010).
} 
Belmont (Nicole). 1975. «L'Académie celtique et Georges Sand. Les débuts des recherches folkloriques en France ». Romantisme, 9, p. 29-38.

Belmont (Nicole). 1995. Aux sources de l'ethnologie française : L'académie celtique. Paris : Éd. du CTHS.

Bénichou (Paul). 1970. Nerval et la chanson folklorique. Paris : Corti.

Béraldi (Henri). 2011. Cent ans aux Pyrénées. Pau : Monhélios.

Bertho (Catherine). 1980. «L'invention de la Bretagne ». Actes de la Recherche en sciences sociales, 35, p. 45-62.

Bertho-Lavenir (Catherine). 1999. La Roue et le stylo : Comment nous sommes devenus touristes. Paris : Odile Jacob. (Le champ médiologique.)

Bödeker (Hans Erich), Veit (Patrice) \& Werner (Michael). 2002. Le Concert et son public : Mutations de la vie musicale en Europe de 1780 à 1914, France, Allemagne, Angleterre. Paris : Éd. de la Maison des Sciences de l'Homme.

Bourguet (Marie-Noëlle). 1989. Déchiffrer la France : La statistique départementale à l'époque napoléonienne. Paris : Éd. des Archives contemporaines.

Boyer (Marc). 2005. Histoire générale du tourisme du XVIe au XXIe siècle. Paris, Budapest, Turin : L'Harmattan.

Briffaud (Serge). 1994. Naissance d'un paysage. La montagne pyrénéenne à la croisée des regards, $X V I^{e}-X X^{e}$ siècles. Toulouse : CIMA-CNRS.

Brunet (J.). 1856. Nouveau guide dans Boulogne-sur-Mer et ses environs, orné d'un plan et de gravures ; suivi d'une notice sur les bains de mer, $4^{e}$ édition. Boulogne-sur-Mer : C. Watel.

Buch (Esteban). 2013. «Le duo de la musique savante et de la musique populaire. Genres, hypergenres et sens commun », p. 43-62, in Théories ordinaires / sous la direction d'Emmanuel Pedler \& Jacques Cheyronnaud. Paris : Éd. de l'EHESS.

Burguière (André). 2003. «L'historiographie des origines de la France. Genèse d'un imaginaire national ». Annales HSS, 58, p. 41-62.

Castanié (F.-M. de). 1857. Guide de Vichy au Mont-Dore par Saint-Nectaire et du Mont-Dore à Clermont-Ferrand, par F. de Castanié. Paris : Chaix.

Chabaud (Gilles), Cohen (Évelyne) \& Coquery (Natacha). 2000. Les Guides imprimés $d u$ XVIe au XXe siècle: Villes, paysages, voyages. Paris : Belin. (Mappemonde.)

Chaline (Jean-Pierre). 1983. «Loisirs et élites sociales : Un exemple normand », p. 185-194, in Oisiveté et Loisirs dans la société occidentale au XIXe siècle / sous la direction d'Ade- line Daumard. Abbeville : Paillart. 
Chambet (Charles-Joseph). 1853. Nouveau guide pittoresque de l'étranger à Lyon. Panorama de la ville et d'une partie de ses environs... par Chambet aîné. 9 é édition. Paris : Maison.

Chaix (Napoléon). 1853. Nouveau guide de Bordeaux aux Pyrénées. Paris : Chaix.

Charles-Dominique (Luc). 2007. Les Musiques traditionnelles en France : Histoire et réalité d'un objet scientifique complexe. Publication en ligne : <http://charlesdominique.wordpress. com/2009/01/04/11-articles-et-7- comptes-rendus-de-luc-charles- dominique/>. Consulté le 2 mars 2013.

Charles-Dominique (Luc). 2008. «L'apport de l'histoire à l'ethnomusicologie de la France », p. 119-154, in L'Ethnomusicologie de la France : De l' «ancienne civilisation paysanne »à la globalisation / sous la direction de Luc Charles-Dominique et Yves Defrance. Paris : L'Harmattan. (Collection Ethnomusicologie et Anthropologie musicale de l'espace français.)

Cheyronnaud (Jacques). 1986. Mémoires en recueils : Jalons pour une histoire des collectes musicales en terrains français. Montpellier : Office départemental d'action culturelle. (Carnets d'ethnologie.)

Cheyronnaud (Jacques). 1997. Instructions pour un recueil général des poésies populaires de la France : 1852-1857. Paris : Éd. du CTHS.

Cohen (Évelyne) \& Toulier (Bernard). 2011. « Les guides de tourisme, un patrimoine et un objet d'étude ». In situ, revue des patrimoines. Publication en ligne : <http://www.insitu. culture.fr/article.xsp?numero=15\&id_article=conclusionguides-0 $>$. Consulté le 2 mars 2013

Cohen (Évelyne). 2011. «Les guides de voyages et de tourisme. Bibliographie indicative 1990$2010 »$. In situ, revue des patrimoines. Publication en ligne : <http://insitu.revues. org/726>. Consulté le 2 mars 2013.

Collet (Paul). 1853. Moûtiers, Brides, Salins : Guide en Tarentaise. Moûtiers : Bocquet.

Corbin (Alain). 2009. L'Avènement des loisirs : 1850-1960. Paris : Flammarion. (Champs Histoire.)

Cousin (Saskia) \& Réau (Bertrand). 2009. Sociologie du tourisme. Paris : La Découverte. (Collection Repères.)

Cousin (Saskia). 2008. «Le tourisme contre les habitants ? , p. 85-96, in Les Constructions locales du politique / sous la direction d'Hélène Bertheleu \& Françoise Bourdarias. Tours : Presses universitaires François-Rabelais. (Perspectives : Villes et territoires.)

Cousin (Saskia). 2011. « De la petite patrie au pays touristique. Jacques- Marie Rougé (18731956) et le "Pays lochois" », p. 167-183, in Initiateurs et Entrepreneurs culturels du tourisme, 1850-1950 / sous la direction de Jean-Yves Andrieux \& Patrick Harismendy. Rennes : Presses universitaires de Rennes.

Csergo (Julia) \& Lemasson (Jean-Pierre). 2008. Voyages en gastronomies : L'invention des capitales et des régions gourmandes. Paris : Autrement. 
Csergo (Julia). 2004. «Parties de campagne. Loisirs périurbains et représentations de la banlieue parisienne, fin XVII ${ }^{\mathrm{e}}-\mathrm{XIX}^{\mathrm{e}}$ siècles ». Sociétés et Représentations, 17, p. 15-50.

Cubero (José). 2009. L’Invention des Pyrénées. Paris : Cairn.

De Certeau (Michel), Julia (Dominique) \& Revel (Jacques). 1993. « La beauté du mort », p. 45-82, in La Culture au pluriel / sous la direction de Michel de Certeau. Paris : Éd. du Seuil. (Points / Essais.) [Première éd. en 1970.]

Fabre (Daniel). 2011. «D'une ethnologie romantique », p. 5-75, in Savoirs romantiques, une naissance de l'ethnologie / sous la direction de Daniel Fabre \& Jean-Marie Privat. Nancy : Presses universitaires de Nancy.

Fierro (Alfred). 1985. «Les guides de voyage au XIXe siècle », t. 3, p. 193, in Le Temps des éditeurs : Du romantisme à la Belle Époque / sous la direction de Henri-Jean Martin et Roger Chartier. Paris : Promodis.

Fouché (Pascal), Péchoin (Daniel) \& Schuwer (Philippe). 2002. Dictionnaire encyclopédique du livre, t. 1. Paris : Éd. du Cercle de la librairie.

Fulcher (Jane F.). 1980. «The Popular Chanson of the Second Empire: "Music of the Peasants" in France ». Acta musicologica, 52, p. 27-37.

Gerbod (Paul). 1983. «Loisirs et santé : Les cures thermales en France (1850-1900) », p. 195205, in Oisiveté et Loisirs dans la société occidentale au XIX ${ }^{e}$ siècle / sous la direction d'Adeline Daumard. Abbeville : Paillart.

Gerbod (Paul). 1985. «Le loisir aristocratique dans les villes d'eaux françaises et allemandes au XIXe siècle (1840-1870) », p. 139-154, in Colloque historique franco-allemand / sous la direction de Karl Ferdinand Werner. Bonn : Röhrscheid.

Gerbod (Paul). 1987. «Le bal en France aux XIX et $^{\mathrm{e}} \mathrm{XX}^{\mathrm{e}}$ siècles », p. 167-173, in Sociabilité, Pouvoirs et Société / sous la direction de Françoise Thelamon. Rouen : Publications de l'université de Rouen.

Gerbod (Paul). 1991. Voyage au pays des mangeurs de grenouilles : La France vue par les Britanniques du XVIII e siècle à nos jours. Paris : Albin Michel.

Gille (Bertrand). 1980. Les Sources statistiques de l'histoire de France : Des enquêtes du XVIIe siècle à 1870. Genève : Droz. [Première éd. en 1964.]

Gimet (François). 1857. Toulouse, son histoire, ses monuments, son musée, son administration, ses plaisirs, ses environs et son commerce, nouveau guide... Toulouse : Gimet.

Guide dans les environs de Paris. 1855. Paris : Paulin et Le Chevalier.

Guide des étrangers aux eaux thermales des Hautes-Pyrénées... 1857. Tarbes : Telmon.

Gritti (Jules). 1967. «Les contenus culturels du guide bleu ». Communications, 10, p. 51-64. 
Guilcher (Goulven). 1994. «Les guides Garnier 1862-1867 ». La Lettre du marché du livre, 39, p. 6-7.

Guilcher (Goulven). 1997a. « Les guides Richard 1824-1836 ». La Lettre du marché du livre, 55, p. 4-7.

Guilcher (Goulven). 1997b. «Les guides Richard 1836-1855 ». La Lettre du marché du livre, 55, p. 6-7.

Guilcher (Goulven). 2011. «Les guides européens et leurs auteurs : Clefs de lecture ». In situ, revue des patrimoines. Publication en ligne : <http:// insitu .revues.org/499>. Consulté le 2 mars 2013.

Guilhou (Adolphe). 1858. Tableaux historiques et descriptifs des Eaux-Bonnes... Guide des malades et des voyageurs. Cahors: Chez tous les libraires.

Jeanpierre (Laurent). 2010. «Frontière», p. 157-169, in Dictionnaire des concepts nomades en sciences humaines / sous la direction d'Olivier Christin. Paris : Métailié.

Joanne (Adolphe). 1856. Les Environs de Paris illustrés : Itinéraire descriptif et historique. Paris : L. Hachette.

Laborde (Denis). 1998. Musiques à l'école. Paris : Bertrand-Lacoste.

Laborde (Denis). 2004. Six études sur la société basque. Paris : L'Harmattan. (Anthropologie du monde occidental.)

Laborde (Denis). 2009. "Écrire une histoire universelle de la musique ? Procédures taxinomiques et aventures encyclopédiques », p. 107-130, in Désirs d'histoire. Politique, mémoire, identité / sous la direction de Denis Laborde. Paris, Turin, Budapest : L'Harmattan. (Anthropologie du monde occidental.)

Lachèse (Eliacin). 1853. Angers ancien et moderne : Guide de l'étranger dans cette ville et ses environs. Angers : De Cosnier et Lachèse.

Lacroix (Jh). 1865. Guide Garnier frères. Nouveau guide général du voyageur aux Pyrénées. Avec des cartes et des vues de villes et de montagnes. Paris : Garnier frères.

Lamothe (Léonce de). 1856. Nouveau guide de l'étranger à Bordeaux et dans le département de la Gironde... par L. L. Bordeaux : P. Chaumas.

Landrin (H.-C. fils). 1853. Guide-Album aux eaux des Pyrénées : Vallée d'Aspe, par H.-C. Landrin, fils... Publié par Jh. Abadie. Tarbes : impr. de T. Telmon.

Lévy (André). 2000. Le Dictionnaire des Pyrénées. Toulouse : Privat.

Millot (Hélène) et al. 2005. La Poésie populaire en France au XIXe siècle : Théories, pratiques et réception. Tus- son : du Lérot. 
Morlier (Hélène). 2007. Les Guides-Joanne : Genèse des Guides-bleus : Itinéraire bibliographique, historique et descriptif de la collection de guides de voyage, 1840-1920. Paris : Éd. Les Sentiers débattus.

Nordman (Daniel). 1986. «Les guides-Joanne, ancêtres des guides bleus », p. 529-567, in Les Lieux de mémoire, La nation. Le territoire, l'État, le patrimoine / sous la direction de Pierre Nora. Paris : Gallimard.

Penez (Jérôme). 2005. Histoire du thermalisme en France au XIXe siècle : Eau, médecine et loisirs. Paris : Impr. Jouve.

Rabault-Mazières (Isabelle). 2000. «Les environs de Paris dans les guides touristiques du XIX ${ }^{\mathrm{e}}$ siècle », p. 317-327, in Les Guides imprimés du XVIe au XXe siècle : Villes, paysages, voyages / sous la direction de Gilles Chabaud, Évelyne Cohen \& Natacha Coquery. Paris : Belin. (Mappemonde.)

Rauch (André). 2001. Vacances en France de 1830 à nos jours. Paris : Hachette littératures. [Première éd. en 1996.]

Réau (Bertrand) \& Poupeau (Franck). 2007. «L'enchantement du monde touristique ». Actes de la Recherche en sciences sociales, 170, p. 4-13.

Soutras (Frédéric). 1858. Guide aux établissements thermaux des Hautes et Basses-Pyrénées et de la Haute-Garonne, suivi d'un coup d'œil rapide sur les thermes de l'Ariège et des PyrénéesOrientales. Bagnères- de-Bigorre : Dossun.

Terquem (Auguste). 1854. Guide du voyageur dans la ville de Metz et ses environs, avec statistique, notes et réflexions sur les institutions, les mœurs, les antiquités... Metz : Lorette.

Thiesse (Anne-Marie). 1997. Ils apprenaient la France : L'exaltation des régions dans le discours patriotique. Paris : Éd. de la Maison des Sciences de l'Homme. (Ethnologie de la France.)

Thiesse (Anne-Marie). 2001. La Création des identités nationales : Europe, XVIIIe-XXe siècle. Paris : Éd. du Seuil. (Points/Histoire.) [Première éd. en 1999.]

Thomas (Jean-Pierre). 1999. Personnalités des Hautes-Pyrénées : Dictionnaire biographique. Aspet : PyréGraph.

Toulier (Bernard). 2010. Villégiature des bords de mer : Architecture et urbanisme : XVIII $X X^{e}$ siècle. Paris : Éd. du patrimoine-Centre des monuments nationaux.

Vajda (Joanne) et al. 2009. «Guides de voyage et lectures urbaines dans l'espace européen (XIX' ${ }^{\mathrm{e}}-\mathrm{XX}^{\mathrm{e}}$ siècles) ». Le Temps des médias, 13, p. 255-261.

Vajda (Joanne). 2010. «Les guides de voyage en Europe (XIX ${ }^{\mathrm{e}}-\mathrm{XX}^{\mathrm{e}}$ siècles), instruments de diffusion d'une culture architecturale ». Sociétés \& Représentations, 30, p. 141-156. 
Venayre (Sylvain). 2012. Panorama du voyage, 1780-1920 : Mots, figures, pratiques. Paris : Les Belles Lettres. (Histoire.)

Villiers (André de). 1855. Nouveau guide complet des promeneurs aux environs de Paris, dans un rayon de 60 kilomètres ; par André de Villiers. Illustré de jolies gravures par Jahyer, et accompagné d'un plan complet. Paris : A. Taride. 